\title{
ОЦЕНКА МАРКЕТИНГОВОЙ НАДЕЖНОСТИ ИННОВАЦИОННЫХ РЕШЕНИЙ (НА ПРИМЕРЕ НЕФТЕПРОМЫСЛОВОГО ОБОРУДОВАНИЯ)
}

\section{(c) 2020 Родионов Дмитрий Григорьевич}

доктор экономических наук, профессор, Высшая инженерно-экономическая школа Санкт-Петербургский политехнический университет Петра Великого, Россия, Санкт-Петербург E-mail: dmitry.rodionov@spbstu.ru

\section{(C) 2020 Конников Евгений Александрович}

кандидат экономических наук, доцент, Высшая инженерно-экономическая школа Санкт-Петербургский политехнический университет Петра Великого, Россия, Санкт-Петербург E-mail: konnikov.evgeniy@gmail.com

\section{(c) 2020 Куракин Владимир Иванович}

соискатель, Высшая инженерно-экономическая школа

Санкт-Петербургский политехнический университет Петра Великого, Россия, Санкт-Петербург

E-mail: v.i.kurakin@yandex.ru

Динамика технологической трансформации практически всех отраслей промышленности экспоненциально возрастает с каждым днем. Следствием данного факта является значимое сокращение цикла инновационного развития технологических решений. В условиях конкурентного рынка все большее значение приобретает прогностические свойства инновационных решений, определяющие их способность удовлетворять потребности потенциальных потребителей. Совокупность данных свойств может быть названа маркетинговой надежностью инновационных решений. При этом, данное свойство крайне многомерно и трудоемко в оценке. В рамках данной статьи предлагается универсальный алгоритм оценки маркетинговой надежности инновационных решений. Оценка маркетинговой надежностью инновационных решений является наиболее значимой для компаний, занимающихся лизингом промышленного оборудования, частным примером которого является нефтепромысловое оборудование.

Ключевые слова: маркетинговая надежность, нефтепромысловое оборудование, лизинг, инновационные решения, информационная среда.

На данный момент мир в полной мере перешёл к шестому технологическому укладу. При этом, необходимо отметить сравнительно более динамичное развитие и интеграцию новых технологических решений. Данный факт в первую очередь обусловлен двумя ключевыми глобальными процессами - глобализацией и цифровизацией. Следствием данных процессов является возможность компьютерного моделирования как отдельных технологических процессов, так и их системной связи, что в свою очередь позволяет оценить перспективность интеграции инновационной технологии на допроизводственном этапе. Таким образом, все большее значение приобретает надежность, реализуемость и универсальность инновационной технологии. Однако, определение данных параметров на ранних этапах развития технологии крайне является крайне трудоемким процессов, а результаты обладают низкой предиктивной значимостью.

Особое значение данная специфика технологического развития оказывает на сферу лизинга. Товарную основу деятельности высокотехнологичного лизингового предприятия составляют инновационные основные средства. При этом инновационные технологические решения являются максимально доступными на ранних этапах рыночной интеграции, что определяет потенциальный финансовый результат лизинговой компании. Однако, как отмечалось ранее, в условиях повсеместной глобализации и цифровизации, процесс рыночной интеграции инновационных технологических решений становится менее долгосрочным. Следствием данного факта является необходимость идентификации потенциально более эффективных 
(перспективных) технологических решений на более ранних этапах, инвестиции в их развитие и поиск потенциальных потребителей на этапе разработки. Таким образом, для современной лизинговой компании все большую роль приобретает аналитическая деятельность, центром которой является поиск, изучение и оценка перспективности инновационных технологических решений.

Описанная специфика характерна не для всех направлений технологического развития. В первую очередь это определяется направлением деятельности компании. Существуют отдельные промышленные направления деятельности, для которых описанная специфика является определяющей. К одному из таких направлений относится нефтяной промысел. Нефтепромысловое оборудование - это комплекс промышленной продукции, предназначенный для использования в нефтегазовом секторе, в который входит техника для бурения, ремонта скважин, геофизических и геологических работ, добычи, транспортировки и переработки углеводородного сырья. Как можно видеть, данный комплекс крайне обширен. При этом, компании, занимающиеся нефтяным промыслом как правило являются полными или частичными монополистами, что в свою очередь определяет относительно низкую емкость рынка, с точки зрения субъектов взаимодействия. При этом оборот в данной крайне значимый, что определяет значительную емкость рынка, с финансовой точки зрения. Данная специфика определяет высокие барьеры входа на рынок лизинга нефтепромыслового оборудования при одновременной необходимости построения стратегически долгосрочных взаимоотношений с потребителем. Следовательно, для компании, заминающейся лизингом нефтепромыслового оборудования, крайне значимым является определение надёжности предлагаемых технологических решений при их единовременном инновационном характере.

Надежность является техническим термином, определяющим свойство объекта сохранять во времени в установленных пределах значения всех параметров, характеризующих способность выполнять требуемые функции в заданных режимах и условиях применения. Однако, в данном случае речь идет об относительной надежности инновационных решений в сфере бурения, ремонта скважин, геофизических и геологических работ, добычи, транспортировки и переработки углеводородного сырья, но не для нефтепромысловых компаний, а для компаний, занимающихся лизингом оборудования, являющегося следствием данных инновационных решений. Для данных компаний наиболее значимыми свойствами данного оборудования является его относительная ликвидность на рынке, способность удовлетворять потребности потребителей (нефтепромысловых компаний), а также высокий уровень инновационной предиктивности (способности данного оборудования на этапе его проектирования и разработки в достаточной мере точно определять финальный результат). Как можно видеть, данные свойства имеют исключительно относительный характер и в первую очередь центрируются в генезисе потребностей потребителей и совокупности решений, направленных на удовлетворение данных потребностей. Таким образом, можно заключить, что исследуемые свойства обладают маркетинговой природой, а не технической. Следовательно, комплексная характеристика, описывающая генезис данных свойств, может быть определена как «маркетинговая надёжность инновационных решений».

Под маркетинговой надежностью инновационных решений можно понимать предиктивное сравнительное свойство инновационных решений сохранять во времени в установленных пределах значения всех параметров, характеризующих способность удовлетворять потребности потенциальных потребителей относительно возможных аналогов. Оценивать данное свойство того или иного инновационного решения необходимо начиная с первичных этапов разработки, сравнивать с возможными аналогами и анализировать динамику изменения. При этом динамическое сравнение необходимо проводить не только с альтернативными инновационными решениями, но и с существующими аналогами. Таким образом, наиболее поверхностный алгоритм оценки маркетинговой надежности инновационного решения может включать в себя следующие этапы:

1. Идентификация конкурентных свойств исследуемого инновационного решения. В случае нефтепромыслового оборудования данные свойства могут быть дифференцированы на технические (совокупность технических параметров, необходимых для поддержания или разви- 
тия существующих технологических процессов), экономические (параметры, отражающие динамическую совокупность затрат ресурсов со стороны потребителя), экологические и нормативные (совокупность параметров, отражающих экологические и нормативные (юридические) последствия использования оборудования, основанного на данном инновационном решении). Также, необходимо отметить возможную необходимость учитывать брэнд-капитал сотрудничества и иную информационную специфику.

2. Идентификация потенциальных и существующих аналогов исследуемого инновационного решения. Обязательным условием включения того или иного решения в перечень альтернатив является возможность оценки всех идентифицированных в рамках первого этапа конкурентных свойств.

3. Калькуляция и сравнительная оценка конкурентных свойств разрабатываемого инновационного решения и его потенциальных и существующих аналогов. В рамках данного этапа производится калькуляция и нормализация идентифицированных ранее свойств. Для целей наглядного сравнения существующих альтернатив может использоваться многоугольник конкурентоспособности.

По результатам оценки осуществляется сравнительный анализ и формулируются выводы относительно маркетинговой надежности исследуемого инновационного решения. Также возможна и калькуляция комплексного показателя маркетинговой надежности исследуемого инновационного решения, что в свою очередь может быть реализовано посредствам взвешенного суммирования значений, определённых ранее свойств, однако, данный подход не позволяет системно проанализировать соотношения между компонентами маркетинговой надежности.

Предлагаемый алгоритм является относительно универсальным, однако обладающим рядом недостатков. В первую очередь, надо отметить необходимость использования эвристических инструментов анализа, что в свою очередь значительно повышает субъективность результирующих выводов, а также повышает затраты реализации, обусловленные привлечением квалифицированных экспертов. Также, данный алгоритм предполагает технологическую специализацию, и разработанные для целей оценки модели могут быть универсализированы исключительно для одного технологического вида инновационных решений. Следовательно, для целей максимизации универсальности алгоритма оценки маркетинговой надежности инновационных решений необходимо дистанцироваться от технических и иных объективных свойств объекта. Данное дистанцированию может быть обеспечена за счет перехода к анализу информационных свойств объекта - инновационного решения.

Сущностно, информационная среда является отражением объективного мира, и первично представляет из себя совокупность динамично транслируемых изменений состояния и взаимодействия объектов объективного мира. Однако, в результате динамично развивающихся процессов глобализации и цифровизации является экспоненциальное развитие информационной интеграции объективного мира. На данный момент значимая часть субъектов генерации информационных потоков находятся в едином цифровом информационном пространстве, скорость трансформации которого настолько высока, что сверх транслируемых изменений объективного мира генерируется множество вторичных и мета-информационных уровней, которые в свою очередь начинают оказывать влияние на объективный мир. Следовательно, взаимодействие объективного мира и информационной среды становится двусторонним и динамическим. Прогностическая специфика информационной среды усложняется отсутствием единого субъекта управления информационными потоками. С другой стороны, данная прогностическая специфика определяется объективность и информационной среды, и ее элементов. Таким образом, сравнительный анализ инновационного решения как объекта информационной среды является потенциально более универсальным и объективным решением в рамках оценки его маркетинговой надежности.

Информационная среда инновационного решения может быть дифференцирована на две базовые компоненты - содержательную и тональную. Содержательная компонента определяется смысловым наполнением элементов информационной среды, посвященных исследуемому инновационному решению. С прикладной точки зрения, содержательная компонента представляет собой совокупность элементарных 
содержательных единиц информационной среды исследуемого инновационного решения и их системную последовательность. Элементарной содержательной единицей является токен, являющийся лексемой (единицей словарного состава языка) или совокупностью лексем. Каждый токен обладает определенной содержательной спецификой, которая может быть конвертирована в условную ценность, относительно инновационного решения. В качестве примера можно рассмотреть содержательную компоненту информационной среды инновационного решения в области 4D сейсмики. 4D сейсмика сущностно является 3D сейсмикой, учитывающие динамические трансформации залежи в процессе разработки. Данная технология на данный момент является инновационной. Содержание информационной среды инновационных решений в области 4D сейсмики в первую очередь характеризуется наличием токенов, описывающих изменения качественных свойств сейсмических исследований, таких как точность, эффективность поиска, успешность при бурении и т.д. Также, содержательной значимостью обладают токены, описывающие экономическую эффективность инновационных решений в данной области. Таким образом, для целей оценки содержательной компоненты информационной среды инновационного решения необходимо определить относительный уровень наличия в нем токенов, описывающих его маркетинговую надёжность. Совокупность данных токенов может быть определена на основе описания конкурентных свойств исследуемого инновационного решения, идентификация которых является первым этапом описанного ранее алгоритма. При этом, также необходимо учитывать динамику данного показателя. Для целей анализа динамики может быть использована классическая методология регрессионного анализа. Распределение оценки содержательной компоненты информационной среды инновационного решения во времени позволит аппроксимировать полученные значения посредствам математической функции, наклон которой в свою очередь отражает скорость прироста, а процент описанной дисперсии - качество аппроксимации, которое может быть использована в качестве удельного веса. Таким образом, определить уровень маркетинговой надежности инновационного решения с точки зрения содержательной компоненты его информационной среды можно посредствам следующей формулы:

$$
\begin{aligned}
& \text { M.R.C.content }=\frac{\overline{t o k_{m \cdot r .} /{ }_{t o k} * T}-\bar{T} * \overline{\text { tok }_{m . r .} / \text { tok }}}{\overline{T^{2}}-(\bar{T})^{2}} * \\
& * \frac{\sum\left(\text { tok }_{\text {m.r. }} /_{\text {tok }}-\overline{\text { tok }_{\text {m.r. }} /_{\text {tok }}}\right)^{2}}{\sum\left(\text { tok }_{\text {m.r. }} /_{\text {tok }}-\overline{\text { tok }_{\text {m.r. } /}{ }_{\text {tok }}}\right)^{2}}
\end{aligned}
$$

Где:

1. to $_{m . r .}$ - число целевых содержательных токенов (токенов входящих в массив, описывающий свойства маркетинговой надежности исследуемого инновационного решения) в общем массиве токенов, сформированном на основе токенизации информационной среды исследуемого инновационного решения.

2. tok - общее число токенов в массиве, сформированном на основе токенизации информационной среды исследуемого инновационного решения.

3. $T$ - номер периода. В качестве периода может использоваться как месяц, квартал или год, так и час или минута. Частота дифференциации определяется спецификой формирования информационной среды исследуемого инновационного решения, в частности скоростью генерации новых информационных элементов.

4. $\overline{\text { tok }_{\text {m.r. }} / \text { tok }}$ - средняя доля целевых содержательных токенов в общем массиве токенов, сформированном на основе токенизации информационной среды исследуемого инновационного решения.

5. to $_{m . r .} /_{\text {tok }}$ - фактическая доля целевых содержательных токенов в общем массиве токенов в конкретный период времени.

6. to $_{\text {m.r. }}$ tok - теоретическая доля целевых содержательных токенов в общем массиве токенов в конкретный период времени, определенная в соответствии с полученным регрессионным уравнением.

Большее значение данного показателя указывает на сравнительно большую маркетинговую надежность исследуемого инновационного решения. Сравнение комплекса альтернатив позволяет идентифицировать наиболее эффективное с точки зрения маркетинговой надежности инновационное решение.

Далее необходимо рассмотреть тональную 
компоненту информационной среды. Данная компонента определяет эмоциональный окрас элементов информационной среды. Данный эмоциональный окрас дифференцируется в соответствии с такими базовыми эмоциями как позитивность, негативность, нейтральность и многими прочимы. На данный момент существует множество автоматизированных инструментов оценки уровня той или иной эмоциональной составляющей в общей тональной компоненте информационной среды. В рамках оценки маркетинговой надежности исследуемого инновационного решения особое значение приобретает соотношение именно уровня позитивности и уровня негативности информационной среды исследуемого инновационного решения. При этом, данный комплексный параметр также необходимо оценивать с учетом динамики изменения, для чего может использовать взвешенное относительно периода времени соотношения уровня позитивной и негативной тональности информационной среды исследуемого инновационного решения. Таким образом, определить уровень маркетинговой надежности инновационного решения с точки зрения тональной компоненты его информационной среды можно посредствам следующей формулы:

$$
M . R . C \cdot \text { tonality }=\sum_{i=1}^{n} \frac{T_{i}}{\sum T} * \frac{\text { ton }_{p_{i}}}{\text { ton }_{n_{i}}}
$$

Где:

1. $n$ - число анализируемых периодов времени.

2. $T_{i}$ - порядковый номер периода $i$.

3. $\Sigma T$ - сумма порядковых номеров анализируемых периодов времени.
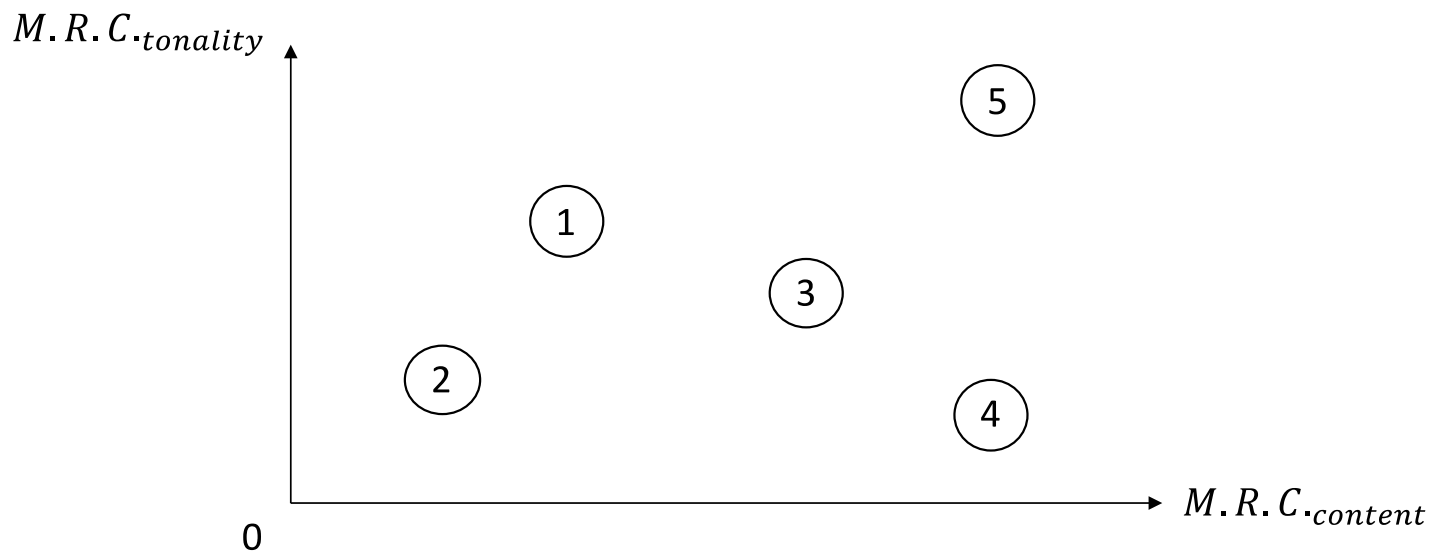

Рисунок 1. Пример карты маркетинговой надежности для пяти альтернативных инновационных решений

4. ton $_{p_{i}}-$ уровень позитивной тональности информационной среды исследуемого инновационного решения в период $i$.

5. $\operatorname{ton}_{n_{i}}-$ уровень негативной тональности информационной среды исследуемого инновационного решения в период $i$.

Значение данного показателя также должно стремиться к сравнительному максимуму. Таким образом, сопоставление значений данных показателей в рамках сравнительного анализа альтернативных инновационных решений, позволяет определить их маркетинговую надежность. Для целей сравнительного анализа может быть использована методология построения карты конкурентов. Пример подобной карты для пяти возможных альтернатив представлен на рисунке 1.

Анализ данного примера позволяет утверждать, что инновационное решение под номером пять обладает сравнительно наибольшим уровнем маркетинговой надежности, в то время как сравнительно наименьшим уровнем обладает решение под номером два. Данный поверхностный анализ позволяет провести сегрегацию возможных альтернативных инновационных решений и далее углублённо анализировать исключительно решение под номером пять, или (при необходимости) также решения под номерами один, три и четыре, так как они обладают сравнительно одинаковым балансом компонент маркетинговой надёжности. Таким образом, приведенная методология анализа информационной среда позволяет трансформировать приведённый ранее алгоритм следующим образом:

1. Идентификация конкурентных свойств исследуемого инновационного решения. Данный первичный этап не отличается от первичного этапа, сформированного ранее алгоритма. 
2. Формирование для каждого конкурентного свойства исследуемого инновационного решения массива целевых токенов. Данные токены должны отражать целевые содержательные характеристики того или иного конкурентного свойства исследуемого инновационного решения. Данные массивы могут формироваться как экспертно, так и на основе токенизации массивов элементов информационной среды (статей, новостных постов и иных текстов), отражающих исследуемую содержательную характеристику.

4. Идентификация потенциальных и существующих аналогов исследуемого инновационного решения. Данный этап также был описан в рамках приведенного ранее алгоритма.

5. Поиск, агрегирование и токеннизация элементов информационной среды как исследуемого инновационного решения, так и его альтернатив. Данный этап может быть автоматизирован посредствам языка программирования Python 3. Более подробно автоматизация данного этапа алгоритма будет рассмотрена в рамках последующих статей. По результатам данного этапа для каждого исследуемого инновационного решения формируется массив токенов.

6. Оценка содержательной и тональной компоненты маркетинговой надежности исследуемых инновационных решений. Данная оценка производится для каждого исследуемого инновационного решения. Методология данной оценки подробно рассмотрена ранее

7. Построение карты маркетинговой надежности для исследуемых инновационных решений и формулирование выводов на основе двумерного сопоставления полученных результатов.

Приведенный алгоритм является значительно более универсальным и требует применения сравнительно меньшего числа эвристических методов анализа. В рамках последующих исследований будет рассмотрена частичная автоматизация данного алгоритма средствами Python 3 и его апробирования в рамках анализа инновационных решений в области нефтепромыслового оборудования.

\section{Библиографический список}

1. Седякина А.А., Конников Е.А. Методика оценки совместимости научно-исследовательского коллектива // Экономические науки. 2020. № 188. С. 77-87.

2. Конников Е.А., Терентьева.Д.А., Конникова О.А. Анализ уровня устойчивого потребления в контексте цифровой информационной среды // Экономические науки. 2020. № 192. С. 114-125.

3. Родионов Д.Г., Ялымов С. В., Конников Е. А. Влияние информационной среды на субъекты малого и среднего предпринимательства // Экономические науки. 2020. № 189. С. 86-91.

4. Родионов Д.Г., Конников Е.А., Алферьев Д.А. Информационный капитал предприятия как целевой показатель развития в рамках цифровых экономических систем // Экономические науки. 2020. № 190. С. $131-137$. 